\title{
Spontaneous Vortex Phase Discovered?
}

\author{
T. K. Ng* and C. M. Varma \\ Bell Laboratories, Lucent Technologies, Murray Hill, New Jersey 07974
}

(Received 29 July 1996)

\begin{abstract}
It is argued that a spontaneous vortex phase probably exists in the recently discovered compound $\mathrm{ErNi}_{2} \mathrm{~B}_{2} \mathrm{C}$ at temperatures below $2.3 \mathrm{~K}$. The consequences of this proposal are discussed. In particular, the magnetic response of the system is studied both above and below $2.3 \mathrm{~K}$, and further experiments are proposed. [S0031-9007(96)02184-9]

PACS numbers: 74.25.Ha, 74.20.De
\end{abstract}

Many years ago it was proposed [1-4] that exotic phases with the coexistence of superconductivity and magnetism may occur in systems with competing superconducting and ferromagnetic components. The analysis was based on the free energy functional $[1,2]$

$$
\begin{aligned}
F=\int d^{3} r[ & \frac{1}{2} a|\psi|^{2}+\frac{1}{4} b|\psi|^{4} \\
& +\frac{\hbar^{2}}{2 m}\left|\left(\nabla-i \frac{2 e}{\hbar c} \vec{A}\right) \psi\right|^{2}+\frac{\vec{B}^{2}}{8 \pi}+\frac{1}{2} \alpha|\vec{M}|^{2} \\
& \left.+\frac{1}{4} \beta|\vec{M}|^{4}+\frac{1}{2} \gamma^{2}|\nabla \vec{M}|^{2}-\vec{B} \cdot \vec{M}\right]
\end{aligned}
$$

where $\vec{B}=\nabla \times \vec{A}, \vec{M}$ is magnetization and $\psi$ is the superconducting order parameter. It was shown that a stable spiral phase, where superconductivity coexists with spiraling magnetization, or a spontaneous vortex phase, where magnetization is more or less uniform in the system but vortices are generated without an external magnetic field, may occur. Subsequently, the spiral phase was discovered in $\mathrm{ErRh}_{4} \mathrm{~B}_{4}$ and $\mathrm{HoMo}_{6} \mathrm{~S}_{8}$ compounds $[5,6]$ in a narrow temperature region between a superconducting phase and a ferromagnetic phase.

More recently, it was discovered that competition between superconductivity and ferromagnetism may occur in a new material $\mathrm{ErNi}_{2} \mathrm{~B}_{2} \mathrm{C}$. We shall show in this paper that $\mathrm{ErNi}_{2} \mathrm{~B}_{2} \mathrm{C}$ is a good candidate for the spontaneous vortex phase, or that the spontaneous vortex phase will become stable under a relatively weak external magnetic field. (We define the spontaneous vortex phase in the presence of a magnetic field as a state where the density of vortices present in the superconductor is larger than that given by the external field.) The consequences of our proposal will be studied. To begin with, we first review some basic features of the Ginsburg-Landau (GL) free energy functional (1) where stability criteria associated with various plausible phases is examined. The analysis of Refs. [1] and [2] are then extended to include the effects of an external magnetic field.

The existence of the spiral and spontaneous vortex phases in $F$ are the direct consequences of the Meissner effect, where a uniform magnetization $\vec{M}$ cannot coexist with a uniform superconducting order parameter $\psi$. For systems with superconducting transition temperature
$T_{c}$ higher than the magnetic transition temperature $T_{m}$, a spiral phase may be stable at temperature $T_{S}$ less than but about $T_{m}$. The wave vector of the spiral is of or$\operatorname{der} Q \sim\left(\lambda_{0} \xi_{M}\right)^{-1 / 2}$, where $\lambda_{0}$ is the penetrating depth of the superconducting component and $\xi_{M} \sim \gamma^{2} / \alpha$ is the coherence length of the magnetic part [2]. The Meissner effect is avoided by having a magnetization whose average is zero on a length scale much smaller than $\lambda_{0}$. At lower temperature, a ferromagnetic state with the superconductivity completely destroyed is usually lower in energy because of the higher energy gain associated with magnetization $\left(\sim k_{B} T_{m}\right)$ compared with the energy gained by superconductivity $\left[\sim\left(k_{B} T_{c}\right)^{2} / E_{F}\right]$. Alternatively, a spontaneous vortex state where magnetization is uniform and the superconducting component exists in the form of a vortex state may be more stable than the ferromagnetic state because of the gain in energy from the superconducting component. However, this state exists only if the internal magnetic field generated by the magnetization $\vec{B}$ satisfies the inequality,

$$
H_{c 1}<B \sim 4 \pi M<H_{c 2},
$$

where $H_{c 1}$ and $H_{c 2}$ are the lower and upper critical magnetic fields associated with $\psi$. For self-generated magnetic fields, the spiral phase is the analog of type I and the spontaneous vortex phase of type II superconductors.

Experimentally, it is found that $\mathrm{ErNi}_{2} \mathrm{~B}_{2} \mathrm{C}$ is superconducting below $10.5 \mathrm{~K}$ [7] and orders antiferromagnetically with a fundamental incommensurate wave vector of $(0.553 a *, 0,0)$ below $6.0 \mathrm{~K}[8] . a^{*}=\pi / a$, where $a$ is the lattice constant in the plane of this tetragonal crystal. The magnetic moments reside mostly on the $\mathrm{Er}^{3+}$ ions which have a measured magnitude $\sim 8 \mu_{B}$ [7]. $M$ vs $H$ measurements indicate that the compound is magnetically strongly anisotropic with the Er magnetic moments essentially along only the in-plane easy axis in (100) and (010) directions [7]. For external magnetic fields significantly larger than $H_{c 1} \sim 500 \mathrm{G}$, the extrapolation of $M(H)$ data back to zero applied field gives a ferromagnetic ordered moment of roughly $0.33 \mu_{B} / \operatorname{Er}$ [9]. Zero-field specific heat measurement also shows a break in the slope of the $C$ vs $T$ curve at $T \sim 2.3 \mathrm{~K}[7,9]$. The existence of the ferromagnetic component in the system is further supported by 
studies on a similar compound $\mathrm{TbNi}_{2} \mathrm{~B}_{2} \mathrm{C}$ which does not manifest superconductivity at $T>2 \mathrm{~K}$, but has a phase transition from antiferromagnetic order to an ordered state with a weakly ferromagnetic component of $0.5 \mu_{B} / T b$ for $T<8 \mathrm{~K} \mathrm{[10]}$. The origin of the incommensurate antiferromagnetic and the transition to the weakly ferromagnetic transition is not quite clear and probably involves both the exchange interactions and the dipolar interactions which are of similar magnitude. Because of the strong easyaxis anisotropy, which forbids smooth deviations from antiferromagnetic states, the incommensurate antiferromagnetic state probably consists of spin domain walls separating antiferromagnetic domains, and the weakly ferromagnetic state is formed by ordering of the domain walls. The distance between domain walls estimated from the incommensurate wave vector is about $19 a$, giving an effective magnetic moment of about $0.42 \mu_{B} / \mathrm{Er}$, which is close to the experimentally zero-field extrapolated value of $0.33 \mu_{B} / \mathrm{Er}$.

It is, in principle, also possible that the ferromagnetic transition at $2.3 \mathrm{~K}$ is due to an impurity phase dispersed in $\mathrm{ErNi}_{2} \mathrm{~B}_{2} \mathrm{C}$ [11]. To account for the observed anisotropy of the magnetization [9], the magnetic axis of the particles of the impurity phase would then have to be aligned in the $a-b$ plane of the host crystal. The condition, $\alpha(T) \leq 4 \pi$ or $T \leq T_{m}$, pertains in this case to the embedded impurity phase. Since the only requirement from Eq. (1) used in this paper for the existence of the spontaneous vortex phase is that a finite uniform magnetization satisfying Eq. (2) exists in the sample, the considerations of Refs. [1] and [2] and of this paper hold also in this case.

At a distance scale much greater than the lattice spacing, the antiferromagnetic component plays a negligible role, and the competition between superconductivity and weak ferromagnetism can be described by a GinsburgLandau functional similar to Eq. (1), except that the $M^{4}$ term must be modified to account for the strong easy-axis anisotropy in this material. The internal magnetic field created by magnetic moment of $0.33 \mu_{B} / \mathrm{Er}$ is approximately $500 \mathrm{G} \sim H_{c 1}$, which is marginal for supporting a spontaneous vortex state. However, a relatively weak magnetic field $\sim H_{c 1}$ should be enough to drive the system from the spiral state into the spontaneous vortex state. In the following we shall investigate this scenario using the GL functional (1). We shall assume that the magnetization $\vec{M}$ lies only in the $x-y$ plane and shall consider external fields only in in-plane directions. The anisotropy in in-plane directions is not included in our analysis. We shall consider $T_{c}>T_{m}$ and shall concentrate on the behavior of the system around $T \sim T_{s}$ which is the regime of experimental interest. The possibility of the system making a second order phase transition to the spiral state at $T=T_{s}$, but driven into spontaneous vortex by an external magnetic field, will be studied. We shall also discuss the alternative possibility of the system making a direct first order transition into the spontaneous vortex state from the superconducting state. First, we consider the temperature region $T>T_{m}$ and study changes in the behavior of the system as $T \rightarrow T_{m}$. In this temperature range $M$ is small, and we can neglect the $M^{4}$ term in the GL functional. The qualitative behavior of the system at this temperature range can be most easily understood by considering the London limit, where $\psi=$ constant, and by neglecting the $|\nabla \vec{M}|^{2}$ term in $F$. It is then easy to minimize $F$ with respect to $\vec{M}$ and $\vec{A}$ to obtain $\vec{M}=\vec{B} / \alpha$, and $\vec{A}=\lambda_{0}^{2}(1-4 \pi / \alpha) \nabla \times \vec{B}$. Putting $\vec{M}$ and $\vec{A}$ back into $F$, we obtain

$$
\begin{aligned}
F \sim \int d^{3} r & \frac{-a^{2}}{2 b}+\left(1-\frac{4 \pi}{\alpha}\right) \frac{1}{8 \pi} \\
& \left.\times\left[\vec{B}^{2}+\left(1-\frac{4 \pi}{\alpha}\right) \lambda_{0}^{2}(\nabla \times \vec{B})^{2}\right]\right\},
\end{aligned}
$$

where $\lambda_{0}^{2}=m c^{2} / 8 \pi e^{2}|\psi|^{2}$ is the London penetration depth for the "pure" superconducting component. $\alpha$ is a decreasing function of temperature, and the magnetic transition (in the absence of the superconducting component) occurs at $\alpha(T)=4 \pi$. Notice that the presence of the magnetic component reduces the overall cost in magnetic energy of the pure superconductor by a factor $(1-4 \pi / \alpha)$. It also reduces the London penetration depth from $\lambda_{0}$ to $\lambda=(\sqrt{1-4 \pi / \alpha}) \lambda_{0}$. The reduction in penetration depth implies that the effective superfluid density observed in the experiment will increase rapidly as $T \rightarrow T_{m}$. As a result, the critical field $H_{c}$ goes down by the same factor $\sqrt{1-4 \pi / \alpha}$. An interesting consequence of the free energy (3) is that the lower critical field $H_{c 1}$ is not much affected by the presence of the magnetic component though the penetrating depth $\lambda$ is strongly reduced as $T \rightarrow T_{m}$. To see that, we consider the superconducting component in the extreme type II limit $\lambda \gg \xi_{0}$. In this limit the energy of creating a vortex line per unit length $\epsilon$ can be computed using the free energy in the London limit (3). For the usual superconductors this quantity is given in the London limit by $\epsilon_{0} \sim\left(\Phi_{0} / 4 \pi \lambda_{0}\right)^{2} \ln \left(\lambda_{0} / \xi_{0}\right)$, where $\Phi_{0}$ is the magnetic flux quantum [12]. In the present case, $\lambda_{0} \rightarrow \lambda$ which enhances $\epsilon$. However, $\epsilon$ is also reduced by the same factor $(1-4 \pi / \alpha)$ because of the overall reduction in free energy (3). As a result, $\epsilon \sim\left(\Phi_{0} / 4 \pi \lambda_{0}\right)^{2} \ln \left(\lambda / \xi_{0}\right)$ in the present case and is only reduced through a reduction in $\lambda$ in the logarithm. Consequently, $H_{c 1}$ is also not much reduced from its clean superconductor value in the presence of the magnetic component. It is interesting to ask what happens in the system when an external magnetic field of order $H_{c 1}$ is applied. For the usual superconductors the density of vortices is of order $\left(2 \pi \lambda_{0}^{2}\right)^{-1}$ when the magnetic field is of order $H_{c 1}$. In the present case, for an external field of order $H_{c 1}$, the external flux supplied in area $2 \pi \lambda^{2}$ is of order $H_{c 1} \times 2 \pi \lambda^{2} \sim \Phi_{0} \times\left(\lambda / \lambda_{0}\right)^{2}$. But the total magnetic field "seen" by the superconductor $\vec{B}=$ 
$\vec{H}+4 \pi \vec{M}=\vec{H} /(1-4 \pi / \alpha) \gg H$ in our approximation, and the total magnetic flux the superconductor sees in area $2 \pi \lambda^{2}$ is of order $\sim$ few $\Phi_{0}$, implying that the density of vortices is of order $\left(2 \pi \lambda^{2}\right)^{-1}$, as in the case of the usual superconductors.

A similar analysis as above can be made when the $|\nabla \vec{M}|^{2}$ term is included in the GL functional. We find that the qualitative behavior of the system is not modified. However, the divergence in $\lambda^{-1}$ as $T \rightarrow T_{m}$ is removed. In particular, the London penetration depth is saturated at a value of order $\lambda \sim\left(\lambda_{0} \xi_{m}\right)^{1 / 2}$ as $T \rightarrow T_{s}$. At temperatures $T$ very close to $T_{s}$, the magnetic response of the system is dominated by the spiral instability. We find that spiraling magnetization develops around the single vortex solution as $T \rightarrow T_{s}$, with the magnitude of the spiral decreasing exponentially with distance away from the vortex core. The decay length of spiral magnetization goes to infinity as $T \rightarrow T_{s}$, signaling the onset of spiral instability. We find also that the energy of the vortex line $\epsilon$ remains finite and is given in the London limit by

$$
\epsilon=\left(\frac{\Phi_{0}}{4 \pi \lambda_{0}}\right)^{2}\left[\ln \frac{\lambda}{\xi}-1+O\left(\frac{\xi_{M}}{\lambda_{0}}\right)\right],
$$

at precisely the spiral instability point where $\lambda=\left(\lambda_{0} \xi_{M}\right)^{1 / 2}$.

The behavior of vortices at $T<T_{s}$ can also be studied in the London limit. In the limit $T \rightarrow T_{s}$, the magnitude of the spiraling magnetization is small, and its effect on vortices can be estimated perturbatively. We find that the single vortex solution is very similar to the solution above the critical temperature, except that the decay length $\lambda_{s}$ for the "extra" spiraling magnetization around the vortex decreases again as $T$ decreases below $T_{s}$, until $\lambda_{s} \sim\left(\lambda_{0} \xi_{M}\right)^{1 / 2}$, where the perturbative solution becomes unreliable. In particular, the energy $\epsilon$ for a single vortex line remains of order $\Phi_{0}^{2} /\left(2 \pi \lambda_{0}^{2}\right)$ throughout the whole temperature range, with no discontinuity across the spiral transition point.

Next, we consider the situation of finite density of vortices and estimate the magnetization as a function of the external magnetic field. Consider the Gibb's energy functional,

$$
G=F-\int d^{3} r \frac{\vec{B} \cdot \vec{H}}{4 \pi},
$$

where the total magnetic field $\vec{B}$ is obtained by minimizing $G$ with respect to $\vec{B}$ and $\vec{M}=(\vec{B}-\vec{H}) / 4 \pi$. First, consider the regime $T>T_{m}$ and the London limit using Eq. (3). Let the applied field strength of order $H_{c 1}$. The total magnetic field $B$ can be obtained easily by comparing the present expression for Gibb's energy, with Gibb's energy for the usual superconductors [12]. We obtain

$$
H \sim\left(1-\frac{4 \pi}{\alpha}\right) B+H_{c 1} \frac{\ln \left(H_{c 2}^{0} / B\right)}{\ln \left(\lambda / \xi_{0}\right)},
$$

where $\quad H_{c 2}^{0} \sim \Phi_{0} /\left(2 \pi \xi_{0}^{2}\right) \quad$ and $\quad M \sim B / \alpha-$ $H_{c 1}\left[\ln \left(H_{c 2}^{0} / B\right) / \ln \left(\lambda / \xi_{0}\right)\right] / 4 \pi$. Notice that the response of the magnetic component $M$ to magnetic field $B$ is almost identical to the response of the pure magnetic system to $B$, except for the correction term $\sim-H_{c 1}$ coming from the Meissner effect. In particular, for a small enough value of $\alpha$, the magnetization may become positive in this range of the magnetic field. The value of $H_{c 2}$ where superconductivity is completely destroyed can also be obtained easily by equating $B=H_{c 2} /(1-4 \pi / \alpha) \sim H_{c 2}^{0}$, obtaining $H_{c 2} \sim(1-4 \pi / \alpha) H_{c 2}^{0}$, indicating that $H_{c 2}$ is reduced in the presence of the magnetic component.

The magnetization curve in the temperature range $T>$ $T_{m}$ thus has the following qualitative features: (1) $M=$ $-H / 4 \pi$ for $H<H_{c 1}$, where the value of $H_{c 1}$ does not depend too strongly on temperature; in particular, there is no singular behavior around the spiral transition. (2) Magnetization starts to increase at $H \sim H_{c 1}$. For $H \sim$ few $H_{c 1}$, the magnetic component already responds to the external magnetic field more or less as if there is no superconducting component in the system. (3) The magnetization continues to increase until it reaches $H=$ $H_{c 2}$, where superconductivity is destroyed. Notice that $M$ may already become positive at magnetic field strength $H \sim$ few $H_{c 1}$.

Next, consider the magnetization curve in the spiral phase. We find that the magnetization curves above and below the spiral transition are qualitatively similar. In particular, the spiral state disappears and is replaced by the spontaneous vortex phase in the external magnetic field of the order of several times $H_{c 1}$. The argument is based on the observation that, in the regime of temperature $T \leq T_{s}$, the vortex solution is not much affected by the spiral component. In particular, the value of $H_{c 1}$ stays more or less the same above and below the spiral transition temperature. As the external magnetic field is of order $H_{c 1}$, the distance between vortices will be of order $\sim \lambda \sim\left(\lambda_{0} \xi_{M}\right)^{1 / 2}$. However, this is of the same order as the period of the spiral state. When the vortex distance is comparable with the period of spiral, the spiral state loses its meaning. Thus we expect that, at this magnetic field range, the spiral state will smoothly cross over to the spontaneous vortex state. In particular, the magnetic response will be similar to that of a pure ferromagnet at temperature $T<T_{m}$, in agreement with what is observed in the $\operatorname{ErNi}_{2} \mathrm{~B}_{2} \mathrm{C}$ compound. The properties of the spontaneous vortex phase can be studied by writing $\vec{M}=\vec{M}_{0}+\vec{M}^{\prime}$, where $M_{0}^{2} \sim(4 \pi-\alpha) / \beta$ is the spontaneous magnetization of the pure ferromagnetic component at temperature $T<$ $T_{m}$, and the GL functional can be expanded to second order in $M^{\prime}$. Neglecting the $|\nabla \vec{M}|^{2}$ term as before, we find $\vec{M}^{\prime}=\left(\vec{B}-4 \pi \vec{M}_{0}\right) /(12 \pi-2 \alpha)$, and the effective GL functional in the London limit in terms of $\vec{B}$ and $\vec{H}$ fields has the same form as (3), except that the total magnetic field $B$ is coupled to an effective external magnetic field $H_{\text {eff }}=4 \pi M_{0}+\eta H$, where $\eta=[(6 \pi-$ $\alpha) /(4 \pi-\alpha)]$. The effective London penetration depth 
is $\lambda=\eta^{-1} \lambda_{0}$, and the criteria for a stable spontaneous vortex state is given by

$$
H_{c 2}>H_{\text {eff }}=4 \pi M_{0}+\eta H \gg H_{c 1} .
$$

Notice that $\lambda$ increases again as the temperature lowers. At very low temperature, $\lambda \rightarrow \lambda_{0}$. In Fig. 1 we show the ratio of total magnetic field to external field $B / H$ as a function of external field $H$ for $H>H_{c 1}$ at several different values of temperatures computed using Eq. (5), with a corresponding equation for $T<T_{m}$. We have chosen $H_{c 2} / H_{c 1}=25.0, \xi_{M} / \xi_{0}=0.5, \alpha(T)=$ $4 \pi+60 \pi\left(T-T_{m}\right)$, and, with saturated magnetization, $4 \pi M=2.0 H_{c 1}$ at zero temperature in generating the figure. It is clear that the zero-field extrapolation of the curve at $T=0.5 T_{m}$ indicates the existence of the ferromagnetic component in the system. Notice that $B / H$ measures the total density of vortices in the system and is $>1$ for $T<T_{m}$. The density of vortices for the pure superconductor is close to the curve with $T=3.0 T_{m}$. The difference arriving from the ferromagnetic component is huge at $T \leq T_{m}$, as can be seen from the figure.

We want to make a few comments on the properties of the spontaneous vortex phase, in particular, in the limit when the saturated magnetic moment is large enough and magnetic anisotropy is strong enough so that a direct first order transition from superconducting phase into spontaneous vortex phase occurs in the absence of external magnetic field. In this case, the effective magnetic field the superconductor sees is always larger than $H_{c 1}$, and there will be no Meissner effect associated with the additional external magnetic field applied on the system, i.e., the effective $H_{c 1}$ of the system is zero and superconductivity "appears" only when vortices are pinned to impurity sites

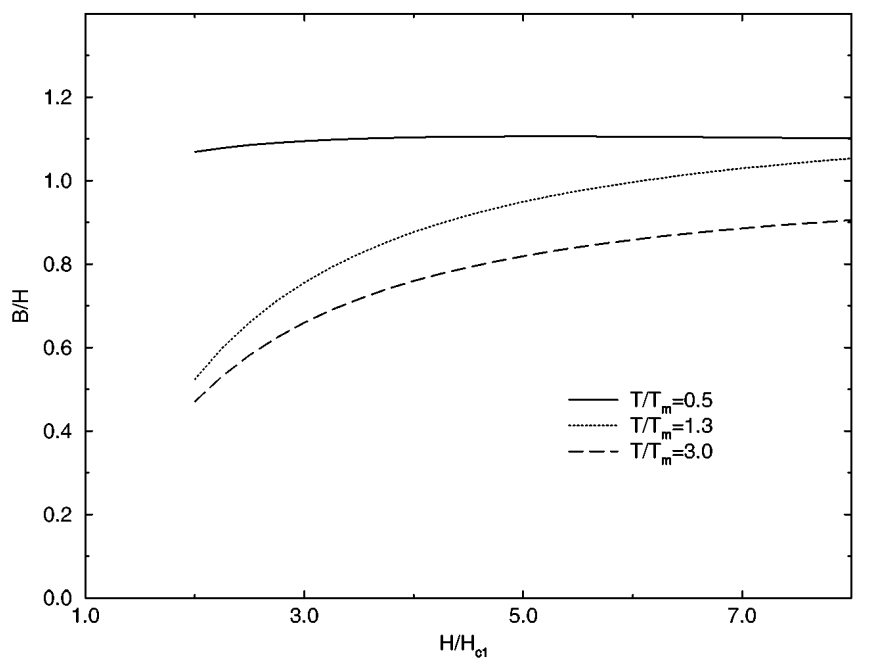

FIG. 1. $B / H$ vs $H / H_{c 1}$ at $H>H_{c 1}$ for several values of temperatures computed approximately in GL theory. The contribution from the ferromagnetic component can be easily deduced from the figure. in the system. Notice that the Meissner effect exists in the spiral phase where $H_{c 1}>0$. Thus measurement of the Meissner effect (for example, by SQUID) will distinguish the spiral and spontaneous vortex phase unambiguously. Experimentally, it seems that the Meissner effect is observed in the $\mathrm{ErNi}_{2} \mathrm{~B}_{2} \mathrm{C}$ compound in the $M$ vs $H$ experiment. However, the experiment is performed in a zero-field cooled environment [7,9], indicating that the result may not reflect the true equilibrium thermodynamic state of the system. Thus the possibility of a zero-field spontaneous vortex phase existing in the compound cannot be ruled out. Direct observation of the spontaneous vortex phase by imaging techniques is suggested.

Recently, neutron scattering experiments [13] have revealed that with a magnetic field applied (nearly) along the $c$ axis, the direction of the vortex-line lattice begins to tilt away from the $c$ axis towards the $a-b$ plane as the temperature is decreased towards the ferromagnetic transition at $T_{m}$. We show [14] in a separate paper that an analysis of Eq. (1) with magnetic anisotropy included leads to this extraordinary behavior as a prelude to the spontaneous vortex phase.

We wish to acknowledge very useful discussions with Paul Canfield, Peter Gammel, and U. Yaron.

*Permanent address: Dept. of Physics, HKUST, Kowloon, Hong Kong.

[1] H. S. Greenside, E. I. Blount, and C. M. Varma, Phys. Rev. Lett. 46, 49 (1981).

[2] E. I. Blount and C. M. Varma, Phys. Rev. Lett. 42, 1079 (1979).

[3] M. Tachiki et. al., Solid State Commun. 31, 927 (1979); 34, 19 (1980).

[4] C. G. Kuper, M. Revzen, and A. Ron, Phys. Rev. Lett. 44, 1545 (1980).

[5] D. C. Moncton et. al., Phys. Rev. Lett. 45, 2060 (1980).

[6] J. W. Lynn et. al., Phys. Rev. Lett. 46, 368 (1981).

[7] B. K. Cho et. al., Phys. Rev. B 52, 3684 (1995).

[8] J. Zarestky et. al., Phys. Rev. B 51, 678 (1995); S. K. Sinha et. al., Phys. Rev. B 51, 681 (1995).

[9] P.C. Canfield, S.L. Bud'ko, and B. K. Cho, Physica (Amsterdam) 262C, 249 (1996).

[10] B. K. Cho et. al., Phys. Rev. B 53, 8499 (1996); P. Dervenagas et. al., Phys. Rev. B 53, 8506 (1996).

[11] In fact, magnetization curves with both the Meissner effect at low fields and a finite extrapolated magnetization at zero field from the data at higher fields have been observed long ago in superconducting samples with added magnetic particles. See, for example, T. H. Alden and J. D. Livingston, J. Appl. Phys. 37, 3551 (1966).

[12] See, for example, M. Tinkham, Introduction to Superconductivity (McGraw-Hill, New York, 1975).

[13] U. Yaron et. al., Nature (London) 82, 236 (1996).

[14] T. K. Ng and C. M. Varma (unpublished). 